\title{
PRESENCIA DE Hippidion principale EN LOS ACANTILADOS COSTEROS DE MAR DEL PLATA (ARGENTINA)
}

\author{
María Teresa ALBERDI', Marcelo ZÁRATE² y \\ José Luis PRADO \\ ' Departamento de Paleobiología, Museo Nacional de Ciencias Naturales, \\ CSIC. José Gutiérrez Abascal, 2. 28006- Madrid, España. \\ ? IANIGLA-CRICYT, C.C. 330, 5500-Mendoza, Argentina. \\ 3 INCUAPA- Departamento de Arqueología, Universidad Nacional del \\ Centro. Del Valle 5737. B7400JWI- Olavarría, Argentina.
}

\begin{abstract}
Alberdi, M.T., Zárate, M. y Prado, J.L. 2001. Presencia de Hippidion principale en los acantilados costeros de Mar del Plata (Argentina). [Hippidion principale from the Mar del Plata sea-cliffs (Argentina).] Revista Española de Paleontología, 16(1), 1-7. ISSN 0213-6937.
\end{abstract}

\begin{abstract}
A complete skull of Hippidion principale, found in Mar del Plata city centre near the beach (Buenos Aires Province, Argentina), is described. Taxonomic identification is mainly based on skull size, morphology and size of the teeth and presence of the dorsal preorbital fosse. Morphological changes due to the fosse presence are also discussed. Paleomagnetic or radiometric dating of the stratigraphical sequence was not available, however, based on stratigraphical correlation the fossiliferous level should be placed between 1.69 and 0.1 Ma, at a Pleistocene age.
\end{abstract}

Keywords: Hippidion principale, Mammalia, Perissodactyla, Pleistocene, Argentina.

\section{RESUMEN}

Se describe un cráneo completo de Hippidion principale hallado en la zona costera de Mar del Plata (provincia de Buenos Aires, Argentina). La descripción está basada principalmente en el tamaño del cráneo, la talla y morfología de sus molares, así como la presencia de la fosa preorbital dorsal y los cambios que su presencia implica en la morfología de la cara. La sección estratigráfica analizada no cuenta con dataciones radiométricas o estudios paleomagnéticos que permitan estimar con cierta precisión la edad de los depósitos portadores de Hippidion. A partir de correlaciones estratigráficas, los restos de Hippidion estarían situados en un lapso que se extiende entre los 1,69 Ma como edad máxima hasta momentos anteriores a Brunhes tardío ( > 0,1 - 0,2 Ma).

Palabras clave: Hippidion principale, Mammalia, Perissodactyla, Pleistoceno, Argentina.

\section{INTRODUCCIÓN}

Los restos analizados en este trabajo, referidos al género Hippidion, provienen de la localidad de Mar del Plata, en la zona costera de la provincia de Buenos Aires y constituyen un nuevo registro de équidos en América del Sur.

Hippidion es un caballo endémico en América del Sur, y su diversificación tuvo lugar en la parte norte de América del Sur o de América Central. Este género representa un grupo homogéneo morfológicamente, y sus distintas especies presentan pequeñas variaciones entre ellas. Alberdi y Prado (1993, 1998) revisaron su situación sistemática y reconocieron tres especies: $H$. saldiasi (Roth), $H$. principale y $H$. devillei (Gervais).

Este hallazgo es el resultado de las tareas de rescate paleontológico que llevó a cabo Alejandro Dondas y el personal del Museo Municipal "Lorenzo Scaglia” de la ciudad de Mar de Plata, a los que clesde aquí queremos dar las gracias. Se exhumó un cráneo completo en los acantilados costeros del sector norte de Mar del Plata (Fig. 1) a unos $4 \mathrm{~km}$ del centro de la ciudad. En la actualidad, los afloramientos no están expuestos, ya que han sido cubiertos por la construcción de defensas costeras. 


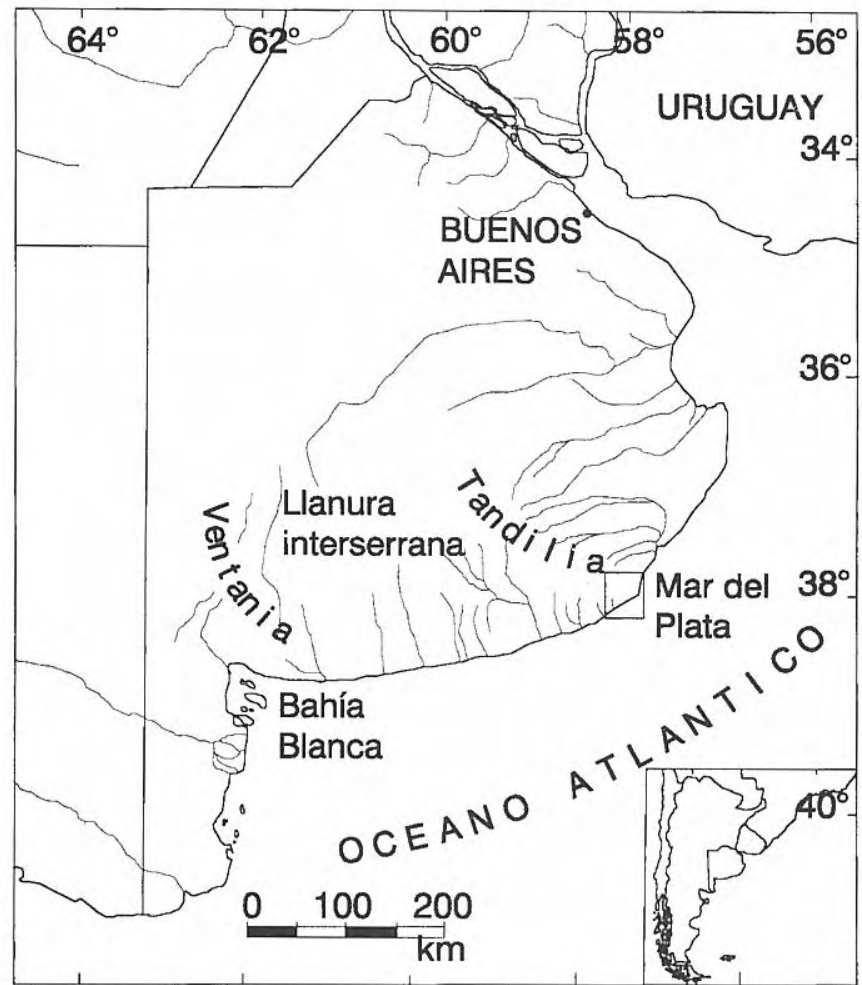

Figura 1. Situación geográfica de la zona de los acantilados costeros del sector norte de Mar del Plata donde se exhumó el cráneo de Hippidion principale descrito en este trabajo.

Geographic situation of the Mar del Plata seacliffs, North sector, where was found the Hippidion principale skull described here.

El objetivo de este trabajo es dar a conocer una descripción detallada de este material, su situación taxonómica precisa y su ubicación estratigráfica.

\section{UBICACIÓN ESTRATIGRÁFICA DEL HALLAZGO}

En el tramo del hallazgo, los acantilados costeros presentan una altura promedio de 6 metros, de los cuales la parte superior $(1,20 \mathrm{~m})$ se encuentra alterada por la acción antrópica resultante de la construcción de la avenida costera. El hallazgo se situó aproximadamente a unos 4 metros de profundidad, en facies fluviales de areniscas muy finas limosas, con niveles diamícticos compuestos por clastos de calcreta; las venas y los tabiques irregulares calcáreos son frecuentes. Exhiben estratificación cruzada, moderada a pobremente definida, con capas de espesores variables entre 20 y $40 \mathrm{~cm}$ que se inclinan unos 2 grados hacia el norte (Fig. 2).

Lateralmente, las facies fluviales presentan geometría lenticular irregular; se apoyan en discordancia erosiva sobre limolitas arcillosas y pasan hacia arriba, a través de contactos netos erosivos, a limolitas arenosas.

Las facies de limolitas arcillosas que afloran con una potencia de unos 2,4 metros en la sección basal del acantilado, están modificadas por pedogénesis, con fisuras y $s$

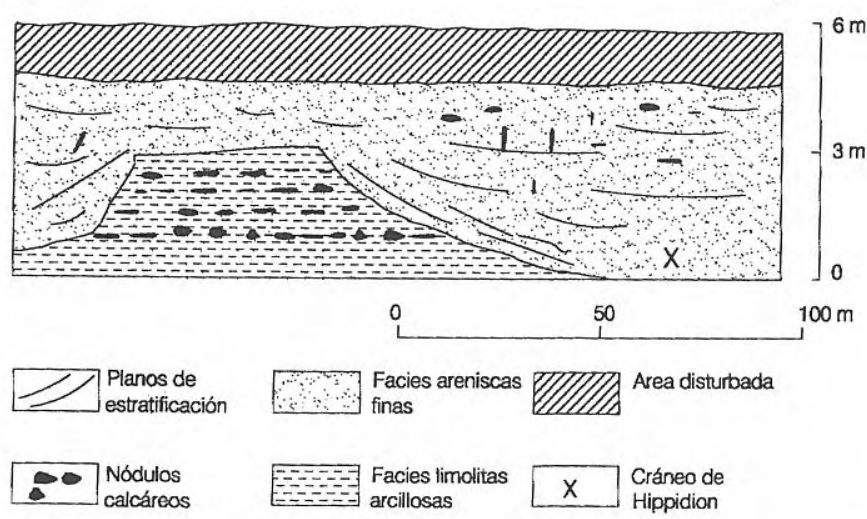

Figura 2. Detalle de la sección estratigráfica de los acantilados costeros del sector norte de Mar del Plata donde se exhumó el cráneo de Hippidion principale descrito en este trabajo.

Stratigraphic section of the Mar del Plata seacliffs, North sector, where was found the Hippidion principale skull described here.

grietas rellenas de carbonato cálcico, coalescentes y formando niveles subhorizontales, lo que otorga un aspecto estratificado al conjunto. Las bioturbaciones de invertebrados, así como las rizoconcreciones y posibles señales de raíces, son muy frecuentes. Estos afloramientos, situados unos 70 metros al sur del sitio del hallazgo, se presentan muy recortados por una superficie de erosión. Las limolitas arenosas que se encuentran sobre las facies arcillosas, presentan un aspecto más homogéneo con estructuras sedimentarias de corriente muy débiles. Incluyen niveles muy delgados de psefitas compuestos por fragmentos de calcreta.

Desde el punto de vista paleoambiental, las facies basales de limolitas arcillosas entoscadas se interpretan como ambientes topográficos bajos con drenaje deficiente, posiblemente vinculados con planicies aluviales. Estas condiciones fueron interrumpidas por un episodio erosivo que elaboró una topografía de relieve muy suave, generadora de valles pandos rellenados por las facies fluviales donde se encontraron los restos de Hippidion. Las limolitas arenosas suprayacentes están vinculadas también con procesos de transporte ácueo que actuaron en el relleno del valle.

\section{CORRELACIÓN Y EDAD DE LOS DEPÓSITOS PORTADORES DE HIPPIDION}

La sección estratigráfica analizada no cuenta con dataciones radiométricas o estudios paleomagnéticos que permitan estimar con cierta precisión la edad de los depósitos portadores de Hippidion. Esta sección está comprendida dentro del tramo Punta Iglesias-arroyo Santa Elena, que Kraglievich (1952) describió en términos muy generales. Según este autor, las formaciones Vorohué (VO) y San Andrés (SA) constituyen la sección basal del frente costero. 
Las unidades mencionadas están compuestas por limos loesoides muy compactos y en parte algo conglomeráticos, en la Formación Vorohué, y concreciones calcáreas numerosas en facies de limolitas arcillosas, en la Formación San Andrés. Por encima se sitúan, según Kraglievich (1952), los depósitos de las formaciones Miramar, Arroyo Seco y Lobería. En consecuencia, y considerando las limitaciones impuestas por la escasa información disponible, utilizamos como criterio de asignación cronológica, la correlación con el tramo de acantilados situado entre Mar del Plata y Miramar, considerado como la secuencia estratigráfica tipo de la región, a partir de la analogía de facies sedimentarias. Así, la unidad basal de limolitas arcillosas es muy probablemente correlacionable con el alomiembro medio de la Aloformación Punta San Andrés (Zárate, 1989), equivalente a los niveles cuspidales de la Formación San Andrés (Kraglievich, 1952), que corresponden al piso-edad Marplatense de Cione y Tonni (1995).

En la sección de Chapadmalal-Miramar, se registran, estratigráficamente por encima, tres niveles originados por transporte ácueo (Zárate, 1989). La posición estratigráfica relativa en la sucesión estratigráfica, el carácter extensivo del ciclo de reactivación reconocido a lo largo de unos $3 \mathrm{~km}$ del frente acantilado en la zona de estudio, así como la naturaleza de las facies fluviales, permiten plantear la correlación tentativa del acontecimiento erosivo que precede a la deposición de las facies fluviales portadoras del resto de Hippidion, con el episodio erosivo que antecede a la acumulación del alomiembro superior de la Aloformación Punta San Andrés, de edad magnética Brunhes (Zárate, 1989). Como hipótesis de correlación alternativa, se plantea la posibilidad de equivalencia con los términos cuspidales del alomiembro medio de la Aloformación Punta San Andrés, de edad magnética Matuyama superior (postOlduvai 1,69 Ma, Zárate 1989), ya que en algunos tramos de la sección de Mar del Plata - Miramar se registraron localmente episodios de reactivación erosiva y facies sedimentarias muy similares aunque de reducida extensión. Dadas las distintas características del paisaje al norte y sur de Mar del Plata, podría haber existido una manifestación diferente del mismo acontecimiento.

En consecuencia, los restos de Hippidion estarían acotados en un lapso que se extiende entre los 1,69 Ma, como edad máxima, hasta momentos anteriores a Brunhes tardío ( > 0,1-0,2 Ma).

\section{MATERIAL Y MÉTODO}

Este cráneo se encuentra depositado en las colecciones del Museo de Ciencias Naturales "Lorenzo Scaglia" de Mar del Plata, Provincia de Buenos Aires (MMP), con la sigla MMP-2600. Lo hemos comparado con otros cráneos de Hippidion procedentes de Tarija (Bolivia), y de la provincia de Buenos Aires (Argentina), depositados en el Museo de la Plata (MLP), Museo Ciencias Naturales "Bernardino Rivadavía" (MCN) y Museo de Ciencias Naturales "Lorenzo
Scaglia" de Mar del Plata (MMP) (ver Alberdi y Prado 1992, 1993).

Para su descripción y análisis osteométrico hemos seguido la nomenclatura y normas recomendadas en la "Hipparion Conference", celebrada en Nueva York en 1981 (Eisenmann et al., 1988), así como los estudios realizados por Alberdi y Prado $(1992,1993)$ sobre este género (Fig. 3). Todas las dimensiones están expresadas en milímetros.

\section{PALEONTOLOGÍA SISTEMÁTICA}

\author{
ORDEN PERISSODACTYLA Owen, 1848 \\ Familia Equidae Gray, 1821 \\ Subfamilia Equinae Gray, 1821 \\ Tribu Equini Gray, 1821 \\ Subtribu Pliohippina Prado y Alberdi, 1996 \\ Género Hippidion Owen, 1869
}
Hippidion principale (Lund, 1845) Fig. 3

\section{Holotipo}

Un $\mathrm{P}^{3-4}$ derecho (Lund lo asignó a un $\mathrm{M}^{2}$ ) de la cueva de Lagoa Santa, del Pleistoceno de Brasil, figurado por Lund (1845: Lámina XLIX, fig. 1). Si bien se dio por perdido (Mones, 1986), está depositado en la Colección Lund del Zoological Museum de Copenhague, con el número 3019, y su procedencia exacta es: "Lapa Come nâo bebe", en la cueva de Lagoa Santa.

\section{Distribución geográfica}

América del Sur, expresamente en distintas localidades de la provincia de Buenos Aires (Argentina, Alberdi y Prado 1992, 1993, 1995), Tarija (Bolivia, Alberdi y Prado, 1993 , 1995), Departamento de Artigas (Uruguay, Ubilla y Alberdi, 1990), en Taguatagua (Chile, Alberdi y Frassinetti, en prensa) y varias localidades de Brasil (Alberdi y Prado 1993, 1995).

\section{Distribución estratigráfica}

Piso - Edad Marplatense al Lujanense (Plioceno superior -Pleistoceno superior).

\section{Diagnosis}

Es un Hippidion de talla grande. El cráneo es significativamente más grande que el de $H$. devillei. La retracción del nasal se sitúa a nivel de $\mathrm{M}^{3}$ o posterior. Las series dentales son significativamente más grandes en longitud P2-M3 que las de $H$. devillei. El esqueleto es el más grande y fuerte de los hipidiformes conocidos, y las extremidades, las más robustas.

\section{Descripción}

Cráneo completo de gran tamaño (MMP-2600), similar al de Hippidion principale (= "Onohippidion muñizi" de Moreno, 1891), con fosa preorbital dorsal grande (DPOF) y profunda (Fig. 3c). No se puede indicar si tiene bolsa posterior ("pocket") o no, debido a que ha perdido la parte interna de la DPOF. No parece que sea doble como ocurría 

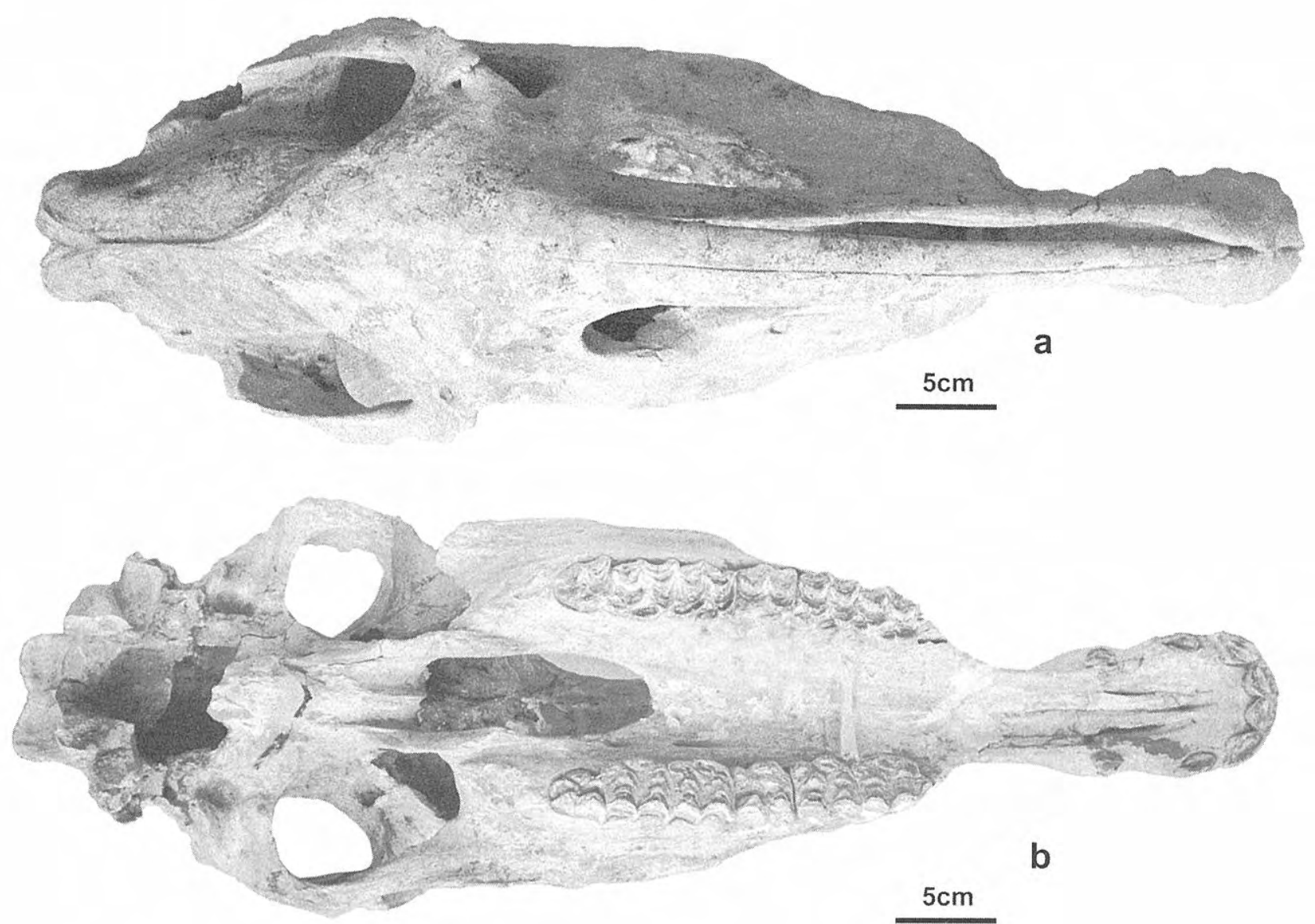

Figura 3. Cráneo completo de Hippidion principale MMP-2600. a, vista dorsal; b, vista ventral; c, vista lateral; d, detalle de los dientes yugales en vista oclusal.

Complete skull of Hippidion principale $M M P-2600$. a, dorsal view; $\boldsymbol{b}$, ventral view; $\boldsymbol{c}$, lateral view; $d$, details of the teeth rows in occlusal view.
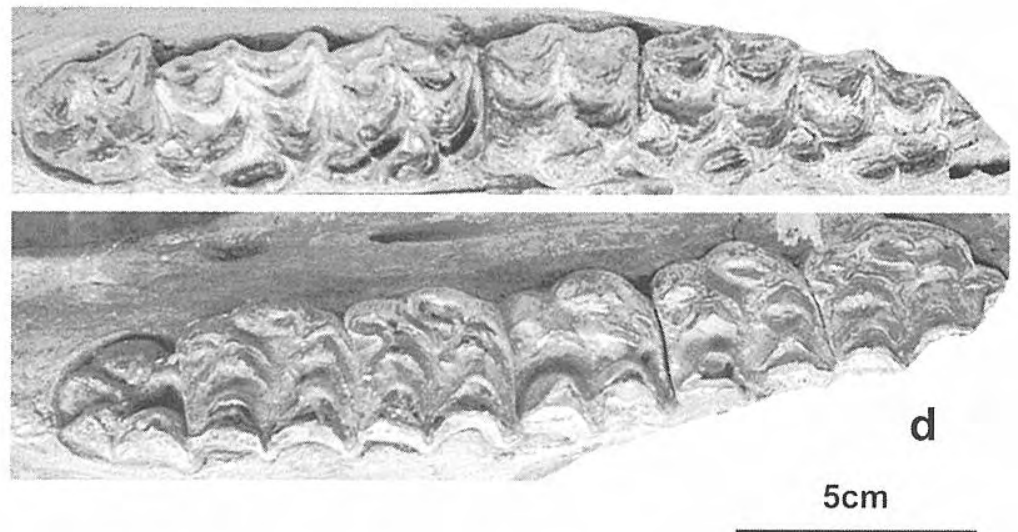


\begin{tabular}{ccccccccccccccccccccc}
\hline sigla & fosa & 1 & 2 & 3 & 4 & 5 & 6 & 7 & 8 & 9 & 10 & 11 & 12 & 13 & 14 & 15 & 16 & 17 & 18 & 19 \\
\hline MLP 6-2 d & con POF & 162 & 102 & 169 & 121 & 290 & 556 & 104 & 85 & 185 & 132 & 44 & 43 & 83,5 & 56 & & 126 & 192 & 211 & 215 \\
MLP 6-2 i & con POF & 148 & 134 & 149 & 123 & 270 & 558 & 105 & 86 & 187 & 112 & 44,5 & 48,5 & 84 & & & 104 & 188 & 191 & 223 \\
1242-TAR & sin POF & 150 & 158 & 126 & 172 & 290 & 533 & 106 & 94,2 & 192 & 111 & 35,9 & 37 & 71,3 & 37 & 65 & 126 & 242 & & \\
MACN 4102 & sin POF & 181,3 & 154 & 154 & 123 & 270 & 600 & 104 & 91,2 & 188,5 & 103 & 44 & 40 & 70 & 42,5 & 63,7 & 114 & 211 & 225 \\
MMP 381 & con POF & & 150 & 146 & 129 & 274 & & 101 & 86,5 & 187,5 & 115 & 40,5 & 48,5 & 79 & & & 137 & 214 & 215 & 219 \\
MACN 401 d & sin POF & 162,5 & 136 & 126 & 132 & 253 & 560 & 99 & 83,4 & 180,7 & 101 & 40,1 & 44,4 & 79,5 & 40,5 & 60,8 & 96 & 191 & 205 & 213 \\
\hline MMP 2600 d & con POF & 165 & 144 & 149 & 143 & 290 & 601 & 104 & 90 & 193 & 117 & 34 & 42 & 77 & 44 & 65 & 93,5 & 206 & 201 & 213 \\
\hline MMP 2600 i & con POF & & & & & & & 106 & 91 & 191,5 & & & & & & & & & \\
\hline
\end{tabular}

\begin{tabular}{|c|c|c|c|c|c|c|c|c|c|c|c|c|c|c|c|c|c|c|c|c|}
\hline sigla & fosa & 20 & 21 & 22 & 23 & 24 & 25 & 26 & 27 & 28 & 29 & 30 & 31 & 32 & 33 & 34 & 35 & 36 & 37 & 38 \\
\hline MLP 6-2 d & con POF & 72 & 137 & 81 & 435 & 225 & 150 & 158 & 16,4 & 66,9 & 57,1 & 360 & 18 & 11 & 123,5 & 54 & 46 & 73 & 29,5 & \\
\hline MLP 6-2 i & con POF & 77,5 & 129 & 79 & 445 & 231 & 145 & 131 & & 70,5 & 57,6 & 369 & 13 & 11 & 123,5 & & 54,5 & 77 & & \\
\hline 1242-TAR & $\sin \mathrm{POF}$ & & 125 & 87 & 495 & 250 & 134 & 145 & 15,9 & 63,5 & 47 & 400 & 28 & & & & & & & \\
\hline MACN 4102 & $\sin \mathrm{POF}$ & 77 & 136 & 77 & 450 & 235 & & 130 & 10 & 63,6 & 58,7 & 370 & 30 & 93 & & & & & & \\
\hline MMP 381 & con POF & 61 & 140 & 82 & & 223 & & 139 & & 70 & 58,5 & & 13 & 19 & 123,3 & & & 68 & & \\
\hline MACN $401 \mathrm{~d}$ & $\sin \mathrm{POF}$ & 72,5 & 133 & 78 & 432 & 218 & 131 & 155 & 19,7 & 67,5 & 53,1 & 334 & 50 & & & & & & 60 & \\
\hline MMP $2600 \mathrm{~d}$ & con POF & 67 & 136 & 77 & 457 & 228 & 134 & & 13.4 & 68 & 60 & 378 & 23 & 33 & 127,2 & 104,5 & 55 & 54,5 & 80,5 & 130 \\
\hline MMP $2600 \mathrm{i}$ & con POF & & & & 460 & 226 & 140 & 116 & & 65 & 54,5 & 380 & 23 & 33,5 & 116,5 & 89 & 45 & 67 & 82 & 130 \\
\hline
\end{tabular}

Tabla 1. Dimensiones del cráneo completo de Hippidion principale MMP-2600, comparado con las dimensiones del cráneo descrito por Moreno (1891), depositado en el Museo de La Plata (MLP 6-2); el cráneo de Tarija (número 1242) depositado en la Universidad de La Paz (Bolivia); el cráneo de Arrecife (MCN-4102) y el de Luján (MCN-401) depositados en el Museo Nacional de Ciencias Naturales "Bernardino Rivadavia"; y el cráneo descrito por Reig (1957 como Onohippidion sp., de Baliza Chica a pocos kilómetros al N de Miramar y depositado en el Museo de Ciencias Naturales "Lorenzo Scaglia" de Mar del Plata MMP-381). Nomenclatura siguiendo las normas recomendadas en la "Hipparion Conference", celebrada en Nueva York en 1981 (Eisenmann et al., 1988), todas las dimensiones están expresadas en milímetros.

Measurements of Hippidion principale skull MMP-2600, compared with: the skull described by Moreno (1891), stored at the Museo de La Plata (MLP 6-2); the skull from Tarija (number 1242) stored at the La Paz University (Bolivia); the skull from Arrecife (MCN-4102), and the skull from Luján (MCN-401), stored at the Museo Nacional de Ciencias Naturales "Bernardino Rivadavia"; and, also, the skull described by Reig (1957) as Onohippidion sp., from Baliza Chica close to N Miramar, stored at the Museo de Ciencias Naturales "Lorenzo Scaglia" of Mar del Plata (MMP-381). The nomenclature follows the recommendations of the "Hipparion Conference", New York, 1981 (Eisenmann et al., 1988). All dimensions are expressed in millimetres.

en la forma descrita por Moreno (1891). La fosa derecha es mayor que la izquierda y el foramen infraorbital está en el borde inferior de la DPOF (la distancia entre la parte posterior de la fosa preorbital y el foramen infraorbital (34) es de $104,5 \mathrm{~mm}$ en el lado derecho y $89 \mathrm{~mm}$ en el izquierdo). La DPOF es más grande en el lado derecho $(33=127,2 / 35=55)$ que en el izquierdo $(116,5 / 45)$. La cavidad orbitaria también es mayor en el lado derecho $(28=68 / 29=60)$ que en el izquierdo $(65 / 54,5)$. La distancia entre la órbita y DPOF es de 33 y $33,5 \mathrm{~mm}$, respectivamente. Sólo en el lado derecho se conserva el meatus auditivo externo, con una altura exterior (27) de 13,4 mm (Fig. 3c). Dimensiones en milímetros ( $\mathrm{mm}$ ) en Tabla 1.

Este espécimen corresponde a un individuo adulto, pero joven. La retracción del nasal alcanza la parte distal de M3 en el lado izquierdo, en el derecho, a nivel del mesostilo de M3. La cresta facial se inicia posteriormente al mesostilo de P4. La anchura basioccipital es aproximadamente de 136 $\mathrm{mm}$ (medida 21, uno de los bordes rotos) y la anchura occipital (20) de $67 \mathrm{~mm}$, mientras que la altura occipital (22) tiene $77 \mathrm{~mm}$.
En vista veniral, la dentición está completa y el desgaste prácticamente sin iniciar sobre $\mathrm{P}^{4}, \mathrm{M}^{2}, \mathrm{y} \mathrm{M}^{3}$, totalmente germen, pero aflora entero (Fig. 3b,d). La morfología es de tipo primitivo, característica del género, aunque en la mayoría no está aún el dibujo bien perfilado. En la serie dental derecha se observa el alvéolo de Pl, en la izquierda no está tan claro. Los dientes yugales están todos recubiertos de abundante cemento. Los premolares (PP) son más grandes que los molares (MM), pero todos son muy robustos y cuadrangulares. El protocono (Pr) oval-alargado, característico, en germen en la mayoría, quedando aislado aún en P4, M2 y M3 derechos y P4 y M3 izquierdos. Figuras iniciadas excepto en P4 sin desgaste, y en M2 y M3 sin desgaste. Hipocono (Hy) muy estrangulado o sin iniciar la figura, en forma de pera con el cuello estrecho, el seno distal entrante y profundo y el lingual bien marcado en P2, P3 y M1. Los pliegues de las fosetas son escasos, las figuras de la superficie oclusal están sólo iniciadas. Los estilos estrechos y bien pronunciados, más el mesostilo que el parastilo.

La sínfisis curvada en su parte anterior, I3 en germen, I2 poco gastado, el diastema entre $\mathrm{I} 3$ y $\mathrm{C}$ no es muy grande $(\sim 20$ 


\begin{tabular}{|c|c|c|c|c|c|c|c|c|}
\hline & $L$ & A & $\mathrm{LPr}$ & $\mathrm{FPr}$ & $\mathrm{Plc}$ & pls fosetas & hipocono & observaciones \\
\hline \multicolumn{9}{|c|}{ serie derecha } \\
\hline $\mathrm{P} 2$ & 40,00 & 28,10 & 10,6 & & & fosetas sin formar & & \\
\hline P3 & 31,80 & 31,00 & 11,1 & oval & 1 & germen & & \\
\hline P4 & 30,60 & 30,00 & 12,5 & & & & & \\
\hline M1 & 30,70 & 31,20 & 11,2 & & 1 & & & \\
\hline M2 & 33,00 & 31,50 & 12,5 & & 1 & & & \\
\hline M3 & 30,00 & 23,00 & germen & & & & & \\
\hline
\end{tabular}

\begin{tabular}{ccccc}
\hline serie izquierda & & & & \\
\hline P2 & 39,70 & 28,50 & 10 & prefoseta abierta \\
P3 & 33,00 & 32,20 & 11 & germinoso \\
P4 & 31,30 & 28,40 & 11,5 & germinoso \\
M1 & 31,30 & 30,50 & 11 & germinoso \\
M2 & 32,30 & 30,30 & 12,2 & germinoso \\
M3 & 28,60 & 23,00 & 10,5 & germinoso \\
\hline
\end{tabular}

Tabla 2. Dimensiones de los dientes yugales del cráneo completo de Hippidion principale MMP-2600, expresadas en milímetros. L, longitud mesio-distal en superficie oclusal; A, anchura buco-lingual en superficie oclusal; LPr, longitud mesio-distal del protocono en superficie oclusal; FPr, forma del protocono en superficie oclusal; Plc, número de pliegues caballinos; pls, pliegues de las fosetas.

Measurements of the cheek teeth of the Hippidion principale skull, MMP-2600, in millimetres. L, occlusal length; A, occlusal breadth; LPr, occlusal length of the protocone; FPr, protocone shape; Plc, pli caballin; pls, enamel plications on the fossettes.

$\mathrm{mm})$. Los caninos son muy robustos y están en germen. La longitud de la serie derecha, incluido P1, es de $203,5 \mathrm{~mm}$. El alvéolo de P1 es de 15/12,3 mm, y la longitud de P1-P4 es de $117 \mathrm{~mm}$. La serie derecha tiene una longitud total de: P2M3 = $193 \mathrm{~mm}(9)$, P2-P4 = $104 \mathrm{~mm}(7)$, M1M3 = $90 \mathrm{~mm}$ (8); la serie izquierda, de 191,5, 106 y $91 \mathrm{~mm}$, respectivamente. Dimensiones de los dientes en Tabla 2. La parte anterior del vomer llega a nivel del mesostilo de M2.

\section{Discusión}

Los caracteres morfológicos dentales de este ejemplar son similares a los de los distintos ejemplares de Hippidion principale de la provincia de Buenos Aires y de Tarija (Alberdi y Prado, 1993, 1998). Las pequeñas diferencias observadas corresponden a la diferencia de edad entre los ejemplares conocidos de $H$. principale y el cráneo objeto de este estudio. El individuo de Mar del Plata es joven, mientras los otros ejemplares son de individuos más viejos, lo cual queda claramente reflejado en la superficie oclusal de los dientes. Las longitudes de las series dentales son más grandes en el ejemplar de Mar del Plata (Tabla 1), lo cual es concordante con lo anterior (recuérdese que en los dientes de Hippidion, y de los équidos hipsodontos en general, la longitud mesio-distal de sus dientes disminuye con la edad). Como vemos en la Tabla 1, la mayoría de las dimensiones son similares, y las mayores diferencias se sitúan en la zona de la DPOF. La longitud total del cráneo que se describe es $601 \mathrm{~mm}$ (6), igual al cráneo de Arrecife (MCN-4102), un poco menor que el ejemplar 1242 de Tarija (este ejemplar está depositado en la Universidad de La Paz), y algo mayor que el de Luján (MCN-401). Estas diferencias también pueden deberse a la edad del ejemplar en estudio, un individuo más joven. La longitud del hocico (1) es de 165 $\mathrm{mm}$ y sus anchuras (14 y 15) 44 y $65 \mathrm{~mm}$, respectivamente; muy similares a las del ejemplar de Luján (162,5, 40,5 y $60,8 \mathrm{~mm}$, respectivamente); el ejemplar de Tarija (1242) tiene 150,37 y $65 \mathrm{~mm}$, respectivamente (Tabla 1 ).

Como hemos visto más arriba, tanto los caracteres morfológicos como las dimensiones del ejemplar que se describe muestran una gran similitud con los restos de Hippdion de mayor talla de la provincia de Buenos Aires y de Tarija, que corresponden a Hippidion principale (ver Alberdi y Prado, 1993). Las diferencias que se observan se deben por una parte a la edad del individuo y por otra a la presencia o no de DPOF, y a las diferencias de forma y tamaño de la misma. La DPOF del ejemplar de Mar del Plata es grande y está bien desarrollada, siendo distinta de la del ejemplar que utilizó Moreno para describir "Onohippidium" munizi (1891: MLP-6.2). Comparado nuestro cráneo con el ejemplar descubierto por Reig en 1954 y descrito por él en 1957 como Onohippidion sp. de la Formación Vorohué (MMP-381 de Mar del Plata), también presenta una dentición más grande en talla. Examinados estos ejemplares, observamos que la DPOF varía entre ellos, estando más desarrollada en el ejemplar MLP 6.2 que en los dos de Mar del Plata (el de la Formación Vorohué corresponde a un cráneo incompleto), en los cuales la fosa no está subdividida y 
no alcanza el tamaño del ejemplar descrito por Moreno (1891). En el ejemplar MMP-381 se observa que la retracción del nasal sobrepasa la parte distal del DPOF, mientras que en el ejemplar de Mar del Plata que nos ocupa, MMP-2600, la retracción del nasal no es tan profunda. En nuestra opinión, esta desigual retracción del nasal debe estar también relacionada con la edad del individuo.

\section{CONCLUSIONES}

Asignamos el ejemplar de Hippidion de Mar del Plata (MMP-2600) a la especie $H$. principale, tanto por sus caracteres morfológicos como por su tamaño.

Se observa, como ya habíamos apuntado en Alberdi y Prado (1998), la variabilidad de la fosa preorbital en este grupo, así como las variaciones entre los distintos ejemplares de $H$. principale debido a la edad de los individuos.

Desde el punto de vista estratigráfico, y con todas las incertidumbres debidas a la situación geológica de este ejemplar que se han apuntado más arriba, creemos que este ejemplar se debe situar, como más antiguo, en el Pleistoceno inferior (en torno a 1,69 Ma) y como más reciente, en el Pleistoceno superior (0,2-0,1 Ma).

\section{AGRADECIMIENTOS}

Queremos agradecer al Museo de Ciencias Naturales "Lorenzo Scaglia" de Mar del Plata, y de manera especial a su director, el Dr. Orlando Scaglia, y al Sr. Alejandro Dondas, que recuperaron el material y nos facilitaron el estudio del ejemplar. Este trabajo ha sido posible gracias al Proyecto de Investigación conjunta con Iberoamerica, (1994-96) y PB94-0071 de la DGICYT, España, así como los subsidios de la SECYT-UNC, PICT97-1166 y PIACONICET al INCUAPA, Argentina.

\section{BIBLIOGRAFÍA}

Alberdi, M.T. y Frassinetti, D. (en prensa). Presencia de Hippidion y Equus (Amerhippus) (Mammalia, Perissodactyla) y su distribución en el Pleistoceno Superior de Chile. Estudios geológicos.

Alberdi, M.T. y Prado, J.L. 1992. El registro de Hippidion Owen, 1869 y Equus (Amerhippus) Hoffstetter, 1950 (Mammalia, Perissodactyla) en América del Sur. Ameghiniana, 29, 265-284.

Alberdi, M.T. and Prado, J.L. 1993. Review of the genus Hippidion Owen, 1869 (Mammalia: Perissodactyla) from the Pleistocene of South America. Zoological Journal Linnean Society, 108, 1-22.

Alberdi, M.T. y Prado, J.L. 1995. Los équidos de América del Sur. In: Evolución biológica y climática de la región pampeana durante los últimos cinco millones de años (Eds. M.T. Alberdi, G. Leone y E.P. Tonni). Monografías del Museo Nacional de Ciencias Naturales, CSIC,
España, 12, 293-308.

Alberdi, M.T. and Prado, J.L. 1998. Comments on: Pleistocene horses from Tarija, Bolivia, and validity of the genus Onohippidium (Mammalia: Equidae) by B.J. MacFadden. Journal of Vertebrate Paleontology, 18, 669-672.

Cione, A. and Tonni, E. 1995. Chronostratigraphy and "Land-Mammal Ages" in the Cenozoic of Southern South America: Principles, practices, and the "Uquian" Problem. Journal of Paleontology, 69, 135-159.

Eisenmann, V., Alberdi, M. T., De Giuli, C. and Staesche, U. 1988. Studying Fossil Horses. E.J. Brill, Leiden, 1-72.

Gray, J.E. 1821. On the natural arrangement of vertebrose animals. London Medical repository, 15, 296-310.

Kraglievich, J.L. 1952. El perfil geológico de Chapadmalal y Miramar, provincia de Buenos Aires. Revista del Museo Municipal de Ciencias Naturales y Tradicionalista de Mar del Plata, 1, 8-37.

Lund, P.W. 1845. Meddlelse af det Udbytte de I 1844 undersögte Knoglehuler Have avgivet til hundskaben om Brasiliens Dyreverden för sidste Jordomvaeltning. Det köngelige Danske Videnskabernes Selskabs naturvidenskabelige og mathematisk Afhandlinger, 12, 57-94.

Mones, A. 1986. Palaeovertebrata Sudamericana. Catálogo sistemático de los Vertebrados fósiles de América del Sur. Courier Forschungsinstitut Senckenberg, 82, 1-625.

Moreno, F.P. 1891. Onohippidium muñizi: Breve noticia sobre los restos fósiles de un género nuevo de la familia de los Équidos conservados en el Museo de La Plata. Revista del Museo de La Plata, 2, 65-71.

Owen, R. 1848. Description of teeth and portions of jaws of two extinct anthracotherioid quadrupeds ... discovered $\ldots$ in the Eocene deposits on the NW coast of the Isle of Wight. Quarterly Journal of Geology Society of London, 4, 103-141.

Owen, R. 1869. On fossil remains of Equines from Central and South America referable to Equus conversidens, Ow., Equus tau, Ow., and Equus arcidens, Ow. Philosophical Transactions of the Royal Society of London, 159, 559-573.

Prado, J.L. and Alberdi, M.T. 1996. A cladistic analysis of the Horses of the tribe Equini. Palaeontology, 39, 663680.

Reig, O. 1957. Un mustélido del género Galictis del Eocuaternario de la provincia de Buenos Aires. Ameghiniana, 1, 33-47.

Ubilla, M. y Alberdi, M.T. 1990. Hippidion sp. (Mammalia, Perissodactyla, Equidae) en sedimentos del Pleistoceno superior del Uruguay (Edad Mamífero Lujanense). Estudios geológicos, 46, 453-464.

Zárate, M. 1989. Estratigrafía y geología del cenozoico tardío aflorante en los acantilados marinos comprendidos entre Playa San Carlos y el Arroyo Chapadmalal, Partido de Gral Pueyrredón, Provincia de Buenos Aires. Tesis FCN y Museo de la Plata, 220 pp. (inédito).

Manuscrito recibido: 11 de febrero, 2000 Manuscrito aceptado: 27 de julio, 2000 